\title{
Synthesis and Characterization of Modified Cellulose Acetate Propionate Nanocomposites via Sol-Gel Process
}

\author{
Patrycja Wojciechowska, ${ }^{1}$ Zenon Foltynowicz, ${ }^{1}$ and Marek Nowicki ${ }^{2}$ \\ ${ }^{1}$ Faculty of Commodity Science, Poznan' University of Economics, Al. Niepodleglosci 10, 61-875 Poznan', Poland \\ ${ }^{2}$ Faculty of Technical Physics, Poznan' University of Technology, Ul. Nieszawska 13A, 60-965 Poznan', Poland \\ Correspondence should be addressed to Patrycja Wojciechowska; p.wojciechowska@ue.poznan.pl
}

Received 27 June 2012; Accepted 5 November 2012

Academic Editor: Carlos Andres Palacio

Copyright (c) 2013 Patrycja Wojciechowska et al. This is an open access article distributed under the Creative Commons Attribution License, which permits unrestricted use, distribution, and reproduction in any medium, provided the original work is properly cited.

\begin{abstract}
In this study novel organic-inorganic hybrid nanocomposites were synthesized from modified cellulose acetate propionate (MCAP) via sol-gel reaction at ambient temperature. The inorganic phase was introduced in situ by hydrolysis-condensation of tetraethoxysilane (TEOS) in different concentrations, under acid catalysis, in the presence of organic polymer dissolved in acetone. The chemical modification of CAP was monitored by infrared spectroscopy (IR). The nanocomposites structure was characterized by IR analysis and solid state ${ }^{29} \mathrm{Si}$ NMR studies. The spectral data revealed that organic and inorganic phases are linked through covalent bound. Surface morphology of the samples and the degree of dispersion of inorganic phase in the polymer matrix were investigated using atomic force microscopy (AFM) and scanning electron microscopy (SEM). The actual incorporation of the inorganic component into the hybrid nanocomposites was deducted from the residual weight according to thermogravimetric analysis (TGA).
\end{abstract}

\section{Introduction}

Organic-inorganic polymer hybrids are rapidly expanding area of research. Such materials offer the opportunity to combine the desirable properties of organic polymers (toughness, elasticity, and formability) with those of inorganic solids (hardness, chemical resistance, and strength). Solgel techniques generating nanosized silica particles within a polymer matrix have been widely used for the preparation of organic-inorganic hybrid nanocomposites. The advantage of sol-gel route is the ability to prepare organic-inorganic nanostructured materials at ambient temperature and the possibility to control morphology of the growing inorganic phase by the subtle control of various reaction conditions. Sol-gel technique involves hydrolysis of the precursor (metal alkoxide) followed by condensation reactions of the resulting hydroxyl groups. Hydrolysis of metal alkoxide and condensation of the hydroxyl groups and residual alkoxyl groups occur simultaneously. Once the hydrolysis reaction has been initiated, condensation takes place parallel to hydrolysis and occurs even if not all of the alkoxyl groups are hydrolyzed.
Considering the nature of the interface between the organic and inorganic phases, hybrid materials can be categorized into two different classes. The first class corresponds to not covalently bound networks of inorganic and organic phases. These hybrids show weak interactions between the polymer matrix and inorganic phase, such as van der Waals, hydrogen bonding, or weak electrostatic interactions, and can be prepared by physical mixing of an organic polymer with a metal alkoxide. In the second class organic and inorganic phases are linked through strong chemical bonds (covalent or ionic). The use of a suitable coupling agent permits an interconnected network to be obtained. In the second class of the hybrid materials one of the most often employed approaches is to use precursors containing organic functional groups capable of polymerization as well as groups which will form the inorganic network [1-3].

Biobased polymers from renewable resources are attracting interest as a substitute for petrochemical based polymers, predominantly due to environmental concerns (growing problem of plastic waste disposal, increased price of crude oil, and global warming) and secondly because of the realization 


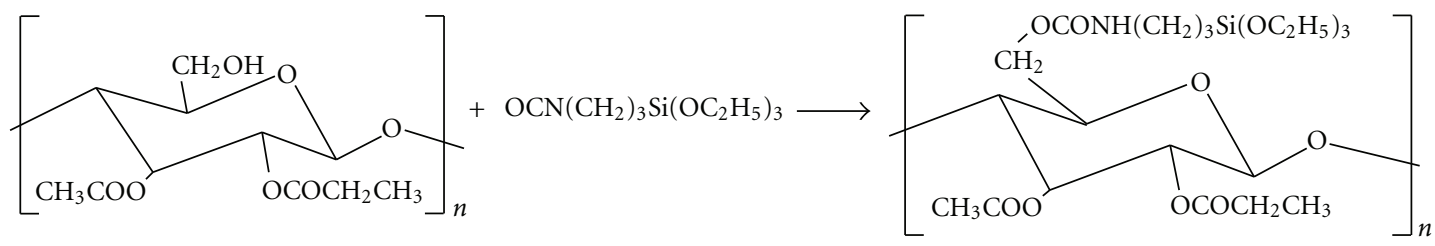

SCHEME 1: Chemical modification of CAP.

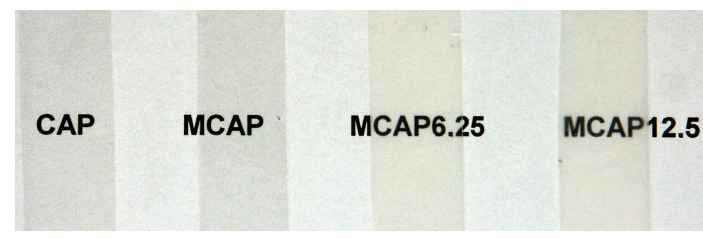

FIGURE 1: Photographs of the samples: CAP, MCAP, MCAP6.25, and MCAP12.5.

that our petroleum resources are finite. Cellulose esters such as cellulose acetate (CA), cellulose acetate propionate (CAP), and cellulose acetate butyrate $(\mathrm{CAB})$ are thermoplastic materials produced through the esterification of the most abundant natural polymer on earth: cellulose. Cellulose plastics are interesting materials for nanocomposites preparation due to their good mechanical properties and processability.

Numerous polymer nanocomposites based on cellulose derivatives have been developed in the past few years [4-10]. In our previous work we reported the preparation of organicinorganic hybrids of the first class (not covalently bound networks of inorganic and organic phases) based on cellulose acetate butyrate (CAB) [11]. In this paper, novel modified cellulose acetate propionate/silica $\left(\mathrm{MCAP} / \mathrm{SiO}_{2}\right)$ nanocomposites were synthesized by sol-gel process applying TEOS as a precursor and ICPTES as a couple agent resulting in the formation of a covalent bound between inorganic phase and polymer matrix. The chemical structure and morphological properties of hybrid nanocomposites are discussed.

\section{Experimental}

2.1. Materials and Reagents. Cellulose acetate propionate (CAP, $M_{n} \approx$ 75000), 3-isocyanatopropyltriethoxysilane (ICPTES, 95\%), dibutyltin dilaurate (95\%), and tetraethoxysilane (TEOS, 98\%) were purchased from Sigma-Aldrich. Dioctyl phthalate (DOP), acetone, tetrahydrofuran (THF), diethyl ether, and hydrochloric acid ( $\mathrm{HCl}, 35 \%-38 \%)$ were purchased from POCH S.A and used as received.

2.2. Modification of Cellulose Acetate Propionate. Cellulose acetate propionate (CAP) was modified through the reaction of 3-isocyanatopropyltriethoxysilane (ICPTES) couple agent with free hydroxyl on the CAP backbone (Scheme 1). The procedure was modeled on the ones reported by Jo and Park [12], and Messori et al. [13]. Functionalization of CAP was performed at a three-necked round-bottom flask equipped with a magnetic stir bar, thermometer, and an argon inlet.

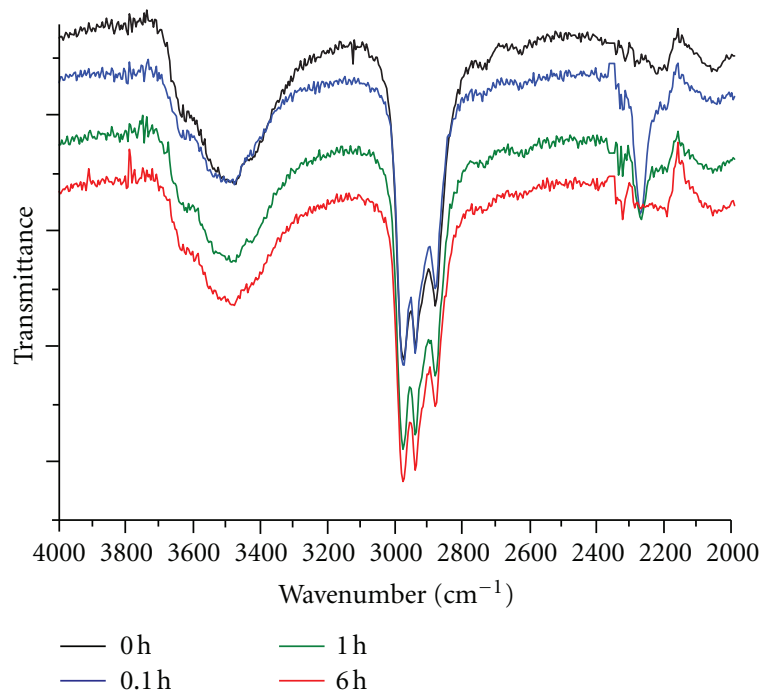

FIGURE 2: IR spectra of the polymer solution during reaction of free hydroxyls on the polymer with ICPTES for different reaction times, showing the disappearance of isocyanate linkage.

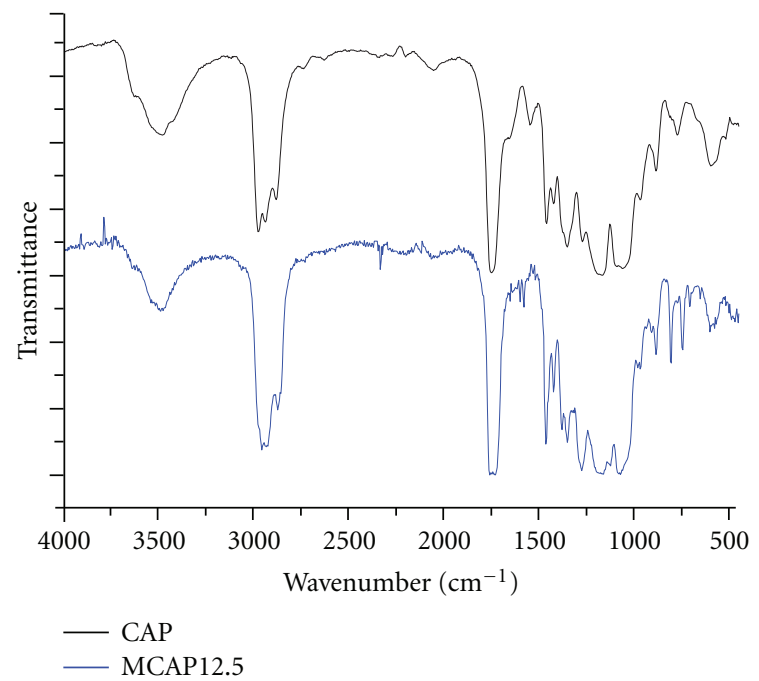

FIGURE 3: IR spectra of CAP, MCAP12.5.

In this flask $13.0 \mathrm{~g}$ of CAP was dissolved in $300 \mathrm{~mL}$ of THF and stirred until a homogenous solution was obtained. To this mixture $1.24 \mathrm{~g}$ of ICPTES in a $10: 1$ polymer/silane molar ratio and $0.085 \mathrm{~g}$ of dibutyltin dilaurate in a $35: 1$ polymer/catalyst molar ratio were added. For this study one 


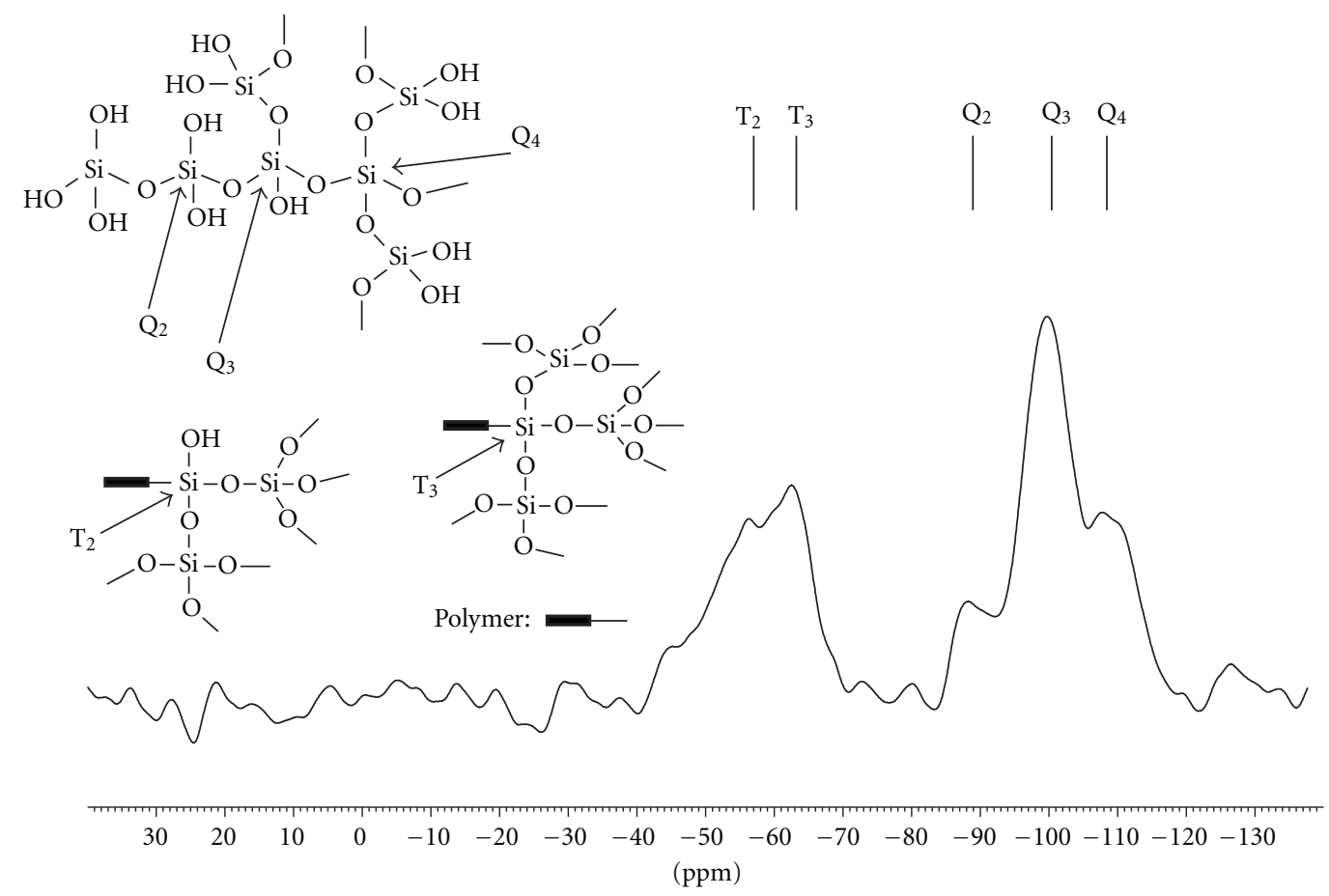

FIGURE 4: MAS ${ }^{29}$ Si NMR spectrum of MCAP12.5.

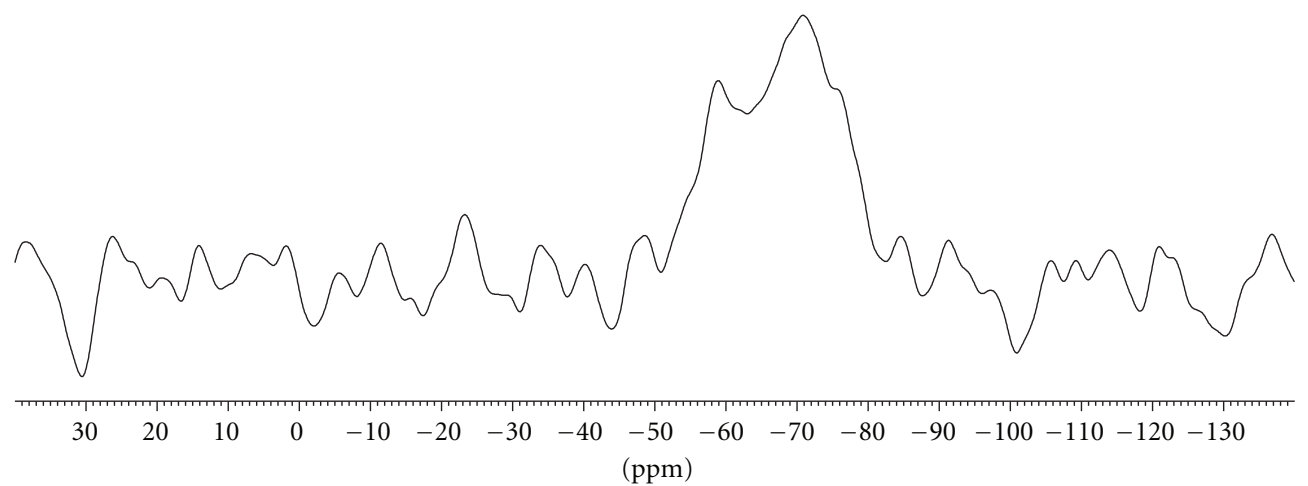

Figure 5: $\mathrm{MAS}^{29} \mathrm{Si}$ NMR spectrum of MCAP.

substituent was grafted per ten CAP repeat units (assuming CAP monomeric molecular weight of 260). The solution was kept under stirring for several hours at $60^{\circ} \mathrm{C}$ until the reaction was complete. The reaction progress was monitored by infrared spectroscopy of the polymer solution at various reaction times. When the reaction was complete, modified polymer (MCAP) was purified by precipitation in diethyl ether and afterwards dried for 24 hours and kept under argon for the preparation of MCAP nanocomposites.

2.3. Preparation of MCAP Nanocomposites. A detailed procedure of organic-inorganic hybrids preparation was described in patent [14]. Sample compositions and characteristics are 


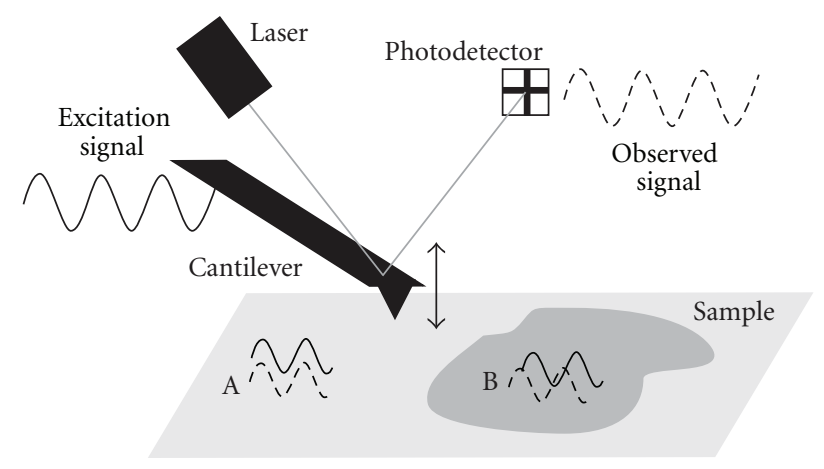

FIGURE 6: Idea of construction "material contrast" image using ICAFM (places A and B have highly different mechanical properties).

TABLE 1: Sample compositions and characteristics.

\begin{tabular}{lccc}
\hline Sample & $\begin{array}{c}\text { Polymer: TEOS } \\
\text { (wt. ratio) }\end{array}$ & $\begin{array}{c}\mathrm{TGA} \\
\mathrm{SiO}_{2} \mathrm{wt} \%\end{array}$ & Nature of the sample \\
\hline $\mathrm{CAP}$ & - & - & transparent \\
MCAP & - & $0.6^{*}$ & translucent \\
MCAP6.25 & $93.75 / 6.25$ & 3 & translucent \\
$\mathrm{MCAP}^{2.5}$ & $87.5 / 12.5$ & 4.8 & translucent \\
${ }^{*} \mathrm{SiO}_{2}$ from ICPTES. & &
\end{tabular}

listed in Table 1. Due to the high brittleness of neat CAP, in order to avoid cracking of the samples, nanocomposites were prepared with $35 \%$ of plasticizer (DOP). A typical preparation of organic-inorganic hybrid was as follows: polymer was placed in a polyethylene beaker and dissolved in acetone. Then DOP and TEOS were added and stirred vigorously until a homogenous solution was obtained. To this mixture catalytic amount of $\mathrm{HCl}(0.1 \mathrm{M})$ was added to initiate the sol-gel process and mixed until it appeared clear and homogenous. The solution was cast in an evaporating PTFE dish and after the solvent removal dried in a vacuum drier at $40^{\circ} \mathrm{C}$ for 12 hours to ensure the complete solvent evaporation. For comparison, a reference sample of neat CAP containing 35\% of plasticizer was also prepared. Figure 1 shows photographs of unmodified CAP and MCAP nanocomposites with thickness in the range of $200-215 \mu \mathrm{m}$. The films' thicknesses were measured using a Sylvac s229 digital indicator equipped with a $10 \mathrm{~mm}$ diameter flat point, with a resolution of $0.001 \mathrm{~mm}$.

2.4. Sample Characterization. IR spectra of the samples were measured with a double-beam spectrophotometer Specord M80 (Carl Zeiss Jena) over a range of $400-4000 \mathrm{~cm}^{-1}$. The ${ }^{29} \mathrm{Si}$ solid-state NMR was performed using a Bruker DSX 300 Avance spectrometer. The spectra of the samples were obtained using cross-polarization and magic-angle spinning (CP/MAS). The spinning rate used was $8 \mathrm{kHz}$. Standard Bruker $4 \mathrm{~mm}$ CPMAS probe was used. Scanning electron microscopy (SEM) analysis was carried out with a Zeiss Evo 40 instrument. Prior to the examination samples were freezefractured and gold-coated. SEM images were taken at the magnification 5000 and 10000. AFM imaging was conducted using easyScan 2 atomic force microscopy (AFM) from Nanosurf. Images were recorded in the tapping mode using PPP-NCLR silicon probes (NanoAndMore $\mathrm{GmbH}$ ). AFM images were collected using Nanosurf Easyscan software and analyzed with Nanotec WSxM [15] computer program. Prior to the AFM analysis samples were twice purified in absolute ethanol using ultrasonic cleaner and mounted onto stainless steel disk with epoxy resin glue. All experiments were carried out at room temperature and ambient humidity. Height, amplitude, and phase images were collected at various scan sizes; maximum $x$ - and $y$-axis range was 10 micrometers. Thermogravimetric analysis (TGA) was carried out using a Shimadzu TGA-50 thermal analyzer. The sample was heated from room temperature to $900^{\circ} \mathrm{C}$ at $10^{\circ} \mathrm{C} / \mathrm{min}$ under the air atmosphere.

\section{Results and Discussion}

3.1. Modification of Cellulose Acetate Propionate. Figure 2 depicts the IR spectra recorded at different reaction times during the synthesis of modified cellulose acetate propionate (MCAP). The progress of the reaction was followed by the decreasing till disappearing of the absorption band related to isocyanate groups at $2272 \mathrm{~cm}^{-1}$ [13]. Under experimental conditions used in this study the modification of CAP was complete within 6 hours. Modified polymer was purified by precipitation in diethyl ether in order to remove the catalyst, soluble in this solvent.

3.2. Chemical Structure of Organic-Inorganic Hybrid Nanocomposites. IR spectroscopy and ${ }^{29} \mathrm{Si}$ NMR were used to study the chemical structure of the synthesized nanocomposites. Figure 3 shows IR spectra of neat polymer (CAP) and nanocomposite (MCAP12.5). The bands around $1000-1100 \mathrm{~cm}^{-1}, 800 \mathrm{~cm}^{-1}$, and $460 \mathrm{~cm}^{-1}$ that appeared in the nanocomposite spectrum indicate that silica is formed via the sol-gel reaction. The peak at $1000-1100 \mathrm{~cm}^{-1}$ can be assigned to asymmetric stretching vibration of the $\mathrm{Si}-\mathrm{O}-\mathrm{Si}$ bonds of the inorganic component. The band at $800 \mathrm{~cm}^{-1}$ is due to symmetric stretching vibration of the $\mathrm{Si}-\mathrm{O}-\mathrm{Si}$ atoms. The presence of the band at $460 \mathrm{~cm}^{-1}$ reveals the existence of $\mathrm{Si}-\mathrm{O}-\mathrm{Si}$ bending mode.

The ${ }^{29} \mathrm{Si}$ solid state NMR spectrum of the MCAP12.5 nanocomposite is shown in Figure 4. The MCAP12.5 sample was prepared by the grafting of ICPTES onto CAP polymer (resulting in T-type structures) followed by the addition of $12.5 \mathrm{wt} \%$ TEOS and sol-gel process (resulting in Q-type structures). Observed in the spectrum $\mathrm{T}^{3}$ and $\mathrm{T}^{2}$ structures are related to trisubstituted siloxane bonds and disubstituted siloxane bonds, respectively. The chemical shifts are -64 and $-58 \mathrm{ppm}$, respectively, and agree closely to the literature values [16]. The Q-type structures observed in the spectrum are related to the in situ formed silica network. According to the literature data very high $Q^{4}$ and $Q^{3}$ structures content but low population of $\mathrm{Q}^{2}$ states suggest a highly intraconnected or cross-linked silicate nanophase. Figure 4 showed very 


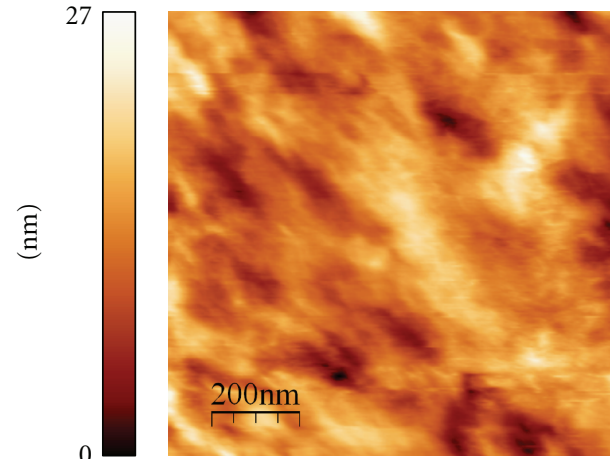

(a)
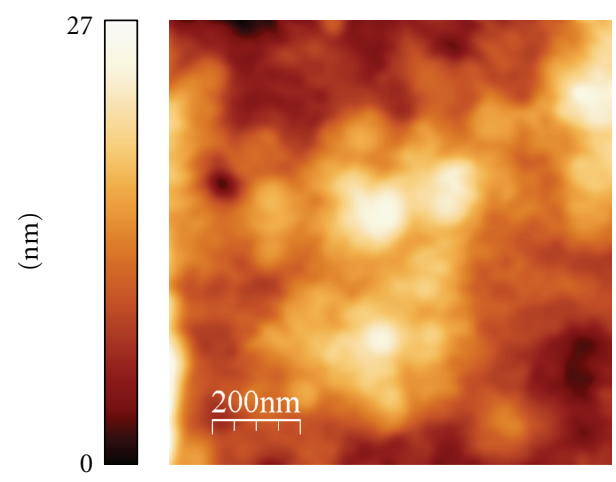

(c)

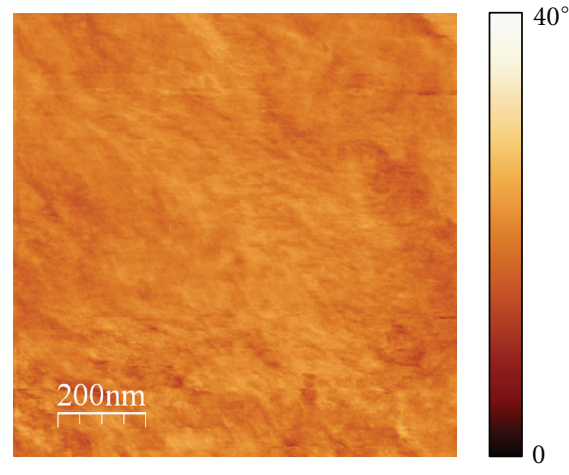

(b)

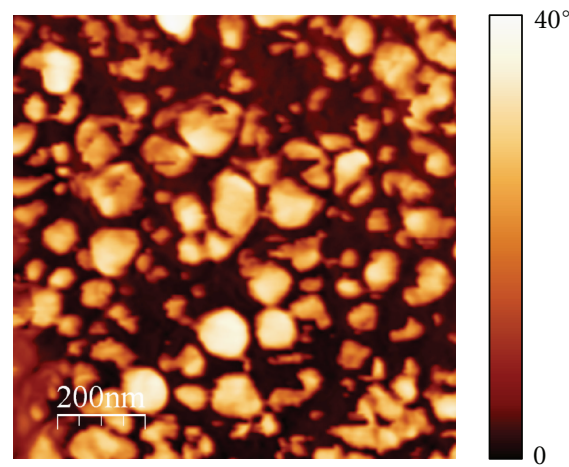

(d)

FIGURE 7: The comparison of AFM images of CAP ((a) topography, and (b) material contrast) and MCAP nanocomposite ((c) topography, and (d) material contrast).

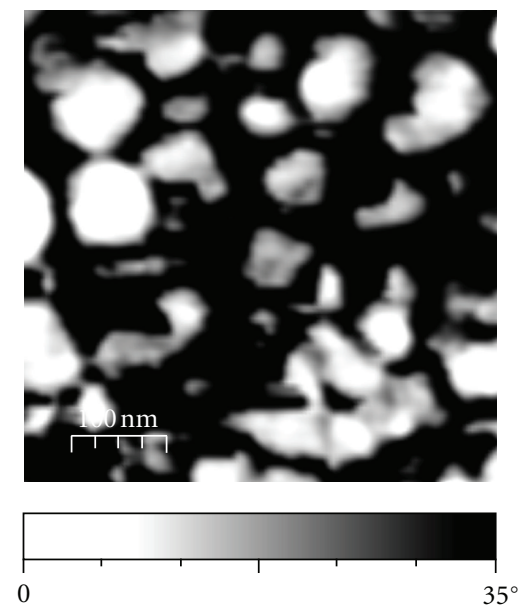

(a)
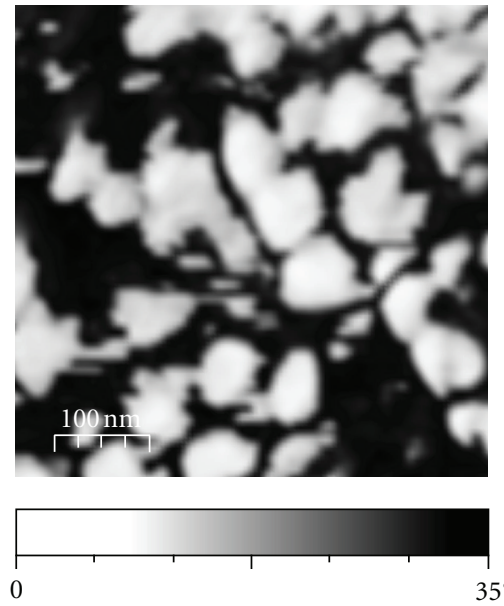

(b)
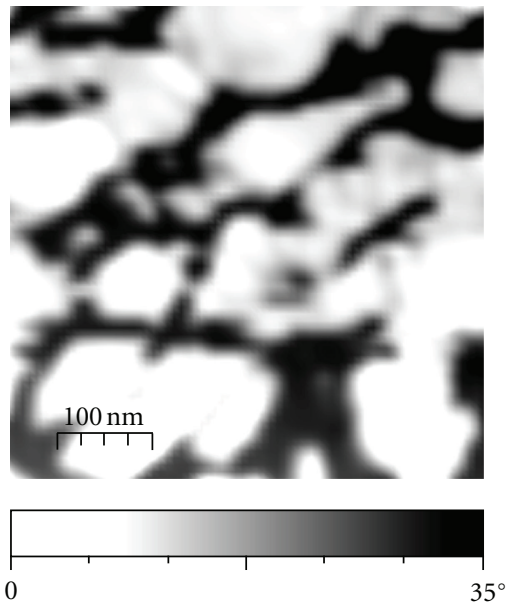

(c)

FIGURE 8: Mechanical contrast images of MCAP (a), MCAP6.25 (b), and MCAP12.5 (c).

high $\mathrm{Q}^{3}$ content (the band at $-102 \mathrm{ppm}$ ), revealing that this is the major structure forming silica network. The $Q^{3}$ structure is assigned to silicon atom connected to the three silicon and one hydrogen atoms or an alkyl group in the second coordination sphere. The peak at $-110 \mathrm{ppm}\left(\mathrm{Q}^{4}\right.$ structure) indicates fully condensed silica. The high intensity of the $\mathrm{Q}^{3}$ band (suggesting the existence of $-\mathrm{OH}$ or $-\mathrm{OR}$ groups) and also $\mathrm{Q}^{2}$ structures observed in the spectra (at $-90 \mathrm{ppm}$ ) indicate that the condensation reaction was incomplete. ${ }^{29} \mathrm{Si}$ NMR spectrum of the MCAP nanocomposite (Figure 5) revealed peaks $\mathrm{T}^{2}$ and $\mathrm{T}^{3}$ observed at -59 and $-71 \mathrm{ppm}$, respectively. The chemical structure of MCAP and 


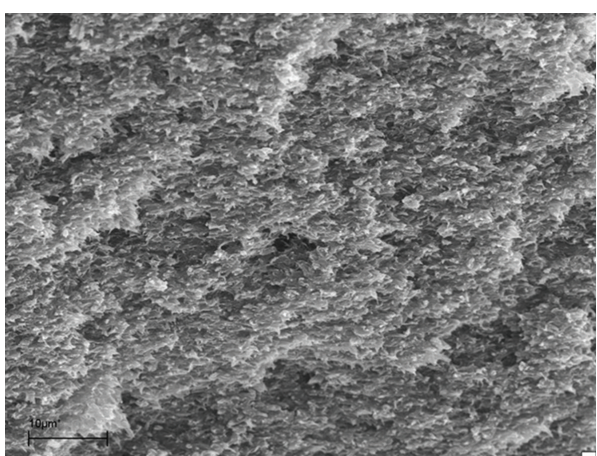

(a)

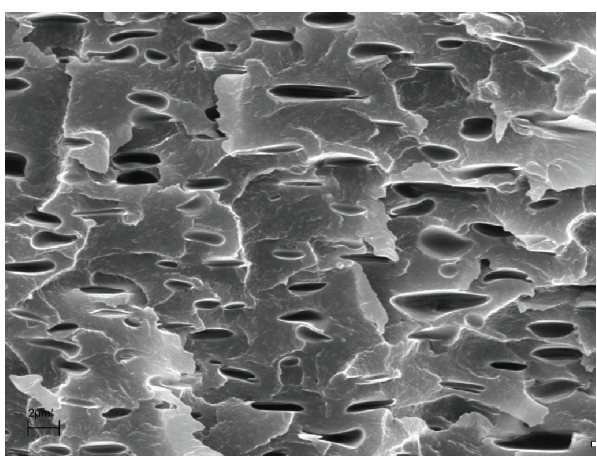

(c)

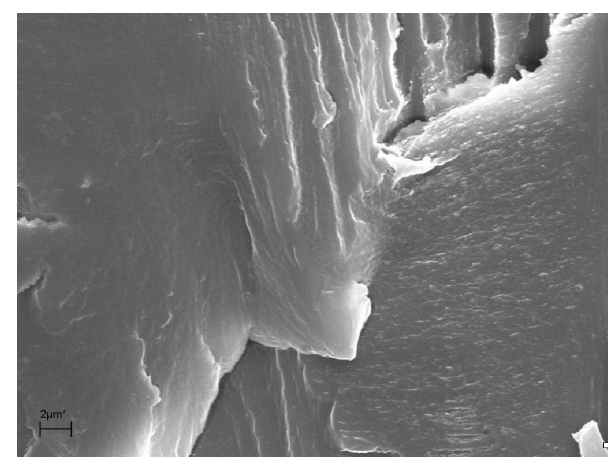

(b)

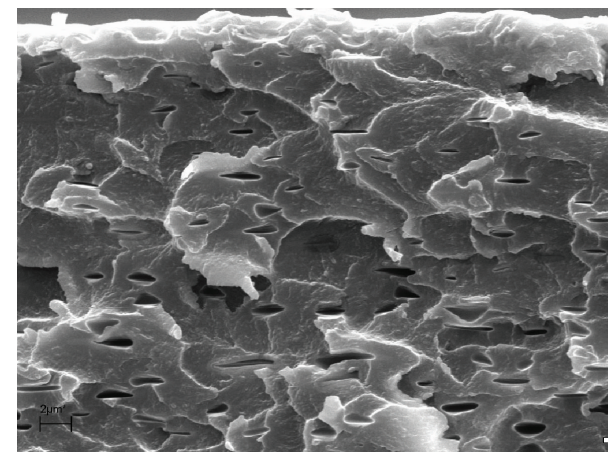

(d)

Figure 9: SEM images: (a) CAP (magnification 5000, scale bar: $10 \mu \mathrm{m}$ ), (b) MCAP nanocomposite (magnification 10000, scale bar: $2 \mu \mathrm{m}$ ), (c) MCAP6.25 nanocomposite (magnification 10000, scale bar: $2 \mu \mathrm{m}$ ), and (d) MCAP12.5 nanocomposite (magnification 10000, scale bar: $2 \mu \mathrm{m})$.

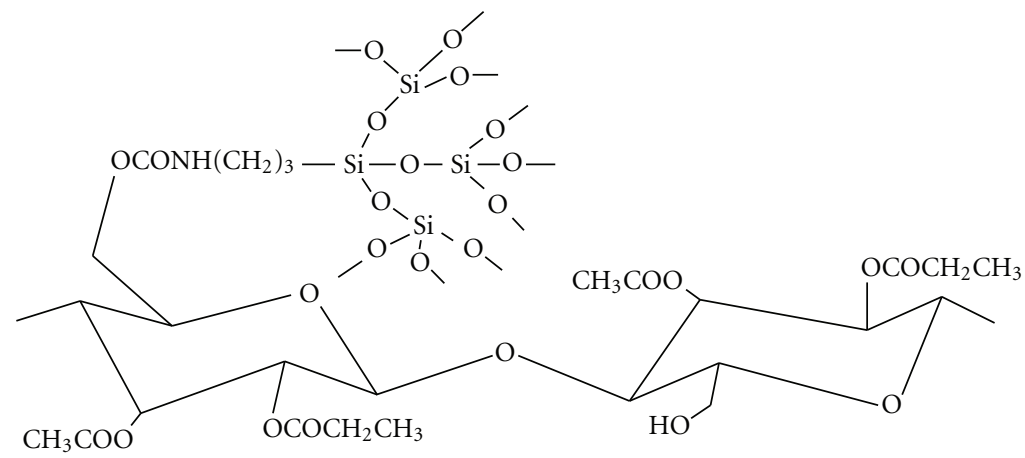

SCHEME 2: Chemical structure of MCAP.

MCAP12.5 nanocomposite is depicted in Schemes 2 and 3, respectively.

\subsection{Morphological Investigation. For surface analysis in-} termittent-contact atomic force microscopy (IC-AFM) was applied. This technique is commonly used for topographical and compositional mapping due to the high spatial and the minimal lateral interaction $[17,18]$. This operation mode is based on cantilever vibrations near the sample surface. In this mode tip strikes the surface with sufficiently large amplitudes to eliminate sticking of the tip to the surface. Due to vibrations, tip-sample surface interaction forces are time dependent, and they contain information on the elastic, viscoelastic, and adhesive properties of the sample. In the IC-AFM mode material composition mapping is performed by recording the shift between the cantilever excitation signal and photodetector signal (compare signals in places A and B on Figure 6) [19]. This shift is known as a "phase signal" and depends on the energy dissipation during the tip-sample interactions [20]. Image build from "phase signal" data is usually called "material contrast" image.

Phase imaging is commonly used to show material contrast in polymer blends and composites. This method is 


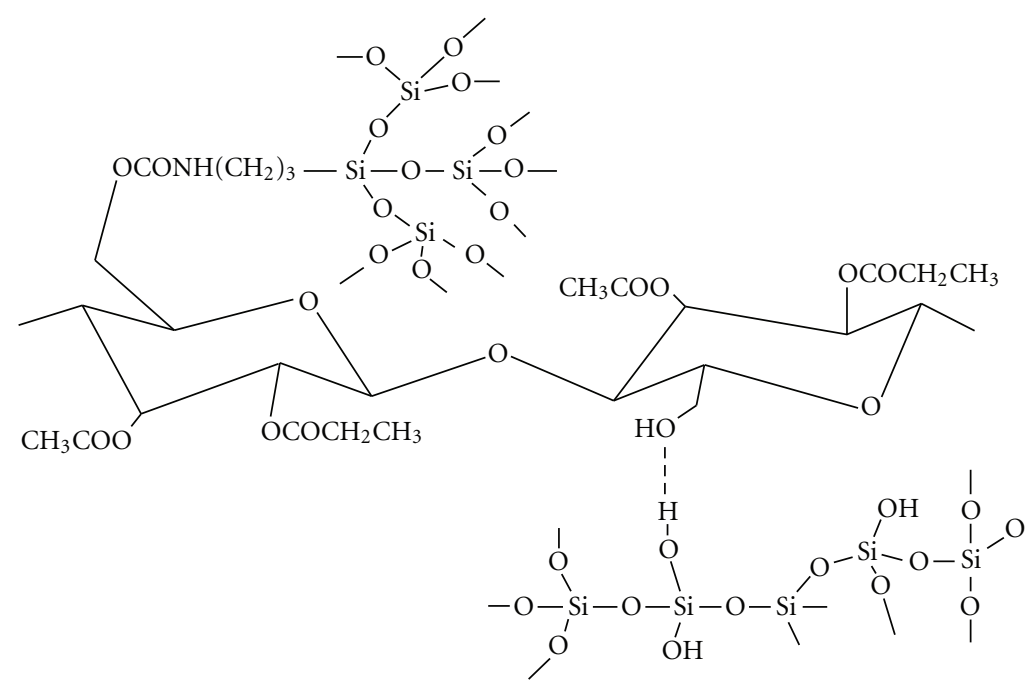

Scheme 3: Chemical structure of MCAP12.5.

very precise and allows detecting structures in composite materials in a nanometer range [21-24].

The presence of two mechanically different phases is clearly seen in the material contrast images. Varied colors displayed on this image show different local mechanical properties (adhesion, Young's modulus, and hardness). This allowed to distinguish modified regions of the polymer matrix, newcreated by the addition of the inorganic component. Figure 7 shows a comparison of the morphology of unmodified CAP with MCAP nanocomposite. Material contrast image of unmodified polymer (CAP) is homogenous (Figure 7(b)) and opposite to nanocomposites based on MCAP does not contain mechanical different areas (Figure $7(\mathrm{~d})$ ). AFM image of MCAP nanocomposite showed that inorganic component modified the mechanical properties of some regions of the polymer matrix. Inorganic phase-rich regions are siloxane network formed during sol-gel process (Schemes 2 and 3).

Figures 8(a), 8(b), and 8(c) show the comparison of MCAP, MCAP6.25, and MCAP12.5 samples, respectively. For the MCAP a region with modified mechanical properties by inorganic phase is about $45 \%$ of the analyzed area (Figure 8(a)). For MCAP6.25 we noted similar structures with diameters between 50 to $150 \mathrm{~nm}$, constituting about $53 \%$ of surface. For MCAP12.5 modified regions are much bigger (ca. 100-300 $\mathrm{nm}$ in length) and frequently connected, covering about $72 \%$ of analyzed surface.

Material contrast images showed clear the presence of inorganic phase-rich regions within polymer matrix, varying with increasing amount of TEOS. Topography images of the samples CAP, MCAP, MCAP6.25, and MCAP12.5 showed RMS roughness: $2.8 \mathrm{~nm}, 2.8 \mathrm{~nm}, 3.4 \mathrm{~nm}$, and $9.5 \mathrm{~nm}$, respectively. The same value of RMS for CAP and for MCAP indicates that chemical modification of cellulose acetate propionate with ICPTES does not impact on surface roughness. On the contrary, the size of inorganic phase-rich regions formed in the sol-gel reaction from varied amount of TEOS considerably changed the RMS roughness of the nanocomposites in comparison with unmodified CAP.
The representative SEM images of unmodified CAP and nanocomposites (Figure 9) revealed that chemical modification of CAP changed the morphological structure of the polymer. The cross-section SEM images of the freezefractured samples revealed that the morphology of unmodified CAP (Figure 9(a)) looks more brittle than of MCAP (Figure 9(b)), MCAP6.25 (Figure 9(c)), and MCAP12.5 (Figure $9(\mathrm{~d})$ ) nanocomposites. SEM images of nanocomposites prepared from MCAP by the addition of 6.25 and 12.5 TEOS (MCAP6.25 and MCAP12.5, resp.) showed cavities (ca. 1.5-4.5 $\mu \mathrm{m}$ in length) formed by evaporation of ethanol evolved during the sol-gel process. Moreover, microscale aggregates of silica particles were not observed, indicating good compatibility of the polymer matrix and covalently bound inorganic phase.

\section{Conclusions}

New hybrid materials with inorganic phase dispersed at the nanometer scale were successfully prepared using modified cellulose acetate propionate via sol-gel process. IR and solid state ${ }^{29} \mathrm{Si}$ NMR spectroscopic studies indicated formation of $\mathrm{Si}-\mathrm{O}-\mathrm{Si}$ links within these materials and showed that inorganic phase is bounded to the CAP matrix through covalent bounds. SEM data revealed different morphological structures of unmodified polymer and $\mathrm{MCAP} / \mathrm{SiO}_{2}$ nanocomposites. AFM studies confirmed growth of inorganic phase network within hybrid materials. AFM data showed the dependence between the amount of TEOS used for nanocomposites preparation and inorganic phase-rich regions size, dispersion, and RMS roughness of the samples.

\section{References}

[1] G. Kickelbick, "Concepts for the incorporation of inorganic building blocks into organic polymers on a nanoscale," Progress in Polymer Science, vol. 28, no. 1, pp. 83-114, 2003. 
[2] S. K. Young, G. C. Gemeinhardt, J. W. Sherman et al., "Covalent and non-covalently coupled polyester-inorganic composite materials," Polymer, vol. 43, no. 23, pp. 6101-6114, 2002.

[3] V. Bounor-Legaré, C. Angelloz, P. Blanc, P. Cassagnau, and A. Michel, "A new route for organic-inorganic hybrid material synthesis through reactive processing without solvent," Polymer, vol. 45, no. 5, pp. 1485-1493, 2004.

[4] M. Yoshioka, K. Takabe, J. Sugiyama, and Y. Nishio, "Newly developed nanocomposites from cellulose acetate/layered silicate/poly( $\varepsilon$-caprolactone): synthesis and morphological characterization," Journal of Wood Science, vol. 52, no. 2, pp. 121-127, 2006.

[5] R. J. B. Pinto, P. A. A. P. Marques, A. M. Barros-Timmons, T. Trindade, and C. P. Neto, "Novel $\mathrm{SiO}_{2}$ /cellulose nanocomposites obtained by in situ synthesis and via polyelectrolytes assembly," Composites Science and Technology, vol. 68, no. 3-4, pp. 1088-1093, 2008.

[6] N. A. Zakharov, Z. A. Ezhova, E. M. Koval, V. T. Kalinnikov, and A. E. Chalykh, "Hydroxyapatite-carboxymethyl cellulose nanocomposite biomaterial," Inorganic Materials, vol. 41, no. 5, pp. 509-515, 2005.

[7] K. Pirkkalainen, K. Leppänen, U. Vainio et al., "Nanocomposites of magnetic cobalt nanoparticles and cellulose," European Physical Journal D, vol. 49, no. 3, pp. 333-342, 2008.

[8] S. Yano, K. Iwata, and K. Kurita, "Physical properties and structure of organic-inorganic hybrid materials produced by sol-gel process," Materials Science and Engineering C, vol. 6, no. 2-3, pp. 75-90, 1998.

[9] F. J. Rodriguez, A. Coloma, M. J. Galotto, A. Guarda, and J. E. Bruna, "Effect of organoclay content and molecular weight on cellulose acetate nanocomposites properties," Polymer Degradation and Stability, vol. 97, no. 10, pp. 1996-2001, 2012.

[10] J. A. de Lima, C. A. Pinotti, M. I. Felisberti, and M. C. Goncalves, "Blends and clay nanocomposites of cellulose acetate and poly(epichlorohydrin)," Composites Part B, vol. 43, no. 5, pp. 2375-2381, 2012.

[11] P. Wojciechowska and Z. Foltynowicz, "Synthesis of organicinorganic hybrids based on cellulose acetate butyrate," Polimery, vol. 54, no. 11-12, pp. 845-848, 2009.

[12] S. Jo and K. Park, "Surface modification using silanated poly(ethylene glycol)s," Biomaterials, vol. 21, no. 6, pp. 605-616, 2000.

[13] M. Messori, M. Toselli, F. Pilati et al., "Flame retarding poly(methyl methacrylate) with nanostructured organicinorganic hybrids coatings," Polymer, vol. 44, no. 16, pp. 4463-4470, 2003.

[14] Polish Patent Office 209829, 2011.

[15] I. Horcas, R. Fernandez, J. M. Gomez-Rodriguez, J. Colchero, J. Gomez-Herrero, and A. M. Baro, "WSXM: a software for scanning probe microscopy and a tool for nanotechnology," Review of Scientific Instruments, vol. 78, no. 1, Article ID 013705, 2007.

[16] S. K. Young, W. L. Jarret, and K. A. Mauritz, "Nafion/ORMOSIL nanocomposites via polymer-in situ sol-gel reactions. 1. Probe of ORMOSIL phase nanostructures by ${ }^{29} \mathrm{Si}$ solid-state NMR spectroscopy," Polymer, vol. 43, no. 8, pp. 2311-2320, 2002.

[17] Q. Zhong, D. Inniss, K. Kjoller, and V. B. Elings, "Fractured polymer/silica fiber surface studied by tapping mode atomic force microscopy," Surface Science, vol. 290, no. 1-2, pp. L688-L692, 1993.
[18] D. Klinov and S. Magonov, "True molecular resolution in tapping-mode atomic force microscopy with high-resolution probes," Applied Physics Letters, vol. 84, no. 14, p. 2697, 2004.

[19] R. Garcia, R. Magerle, and R. Perez, "Nanoscale compositional mapping with gentle forces," Nature Materials, vol. 6, pp. 405-411, 2007.

[20] J. P. Cleveland, B. Anczykowski, A. E. Schmid, and V. B. Elings, "Energy dissipation in tapping-mode atomic force microscopy," Applied Physics Letters, vol. 72, no. 20, pp. 2613-2615, 1998.

[21] P. Achalla, J. McCormick, T. Hodge et al., "Characterization of elastomeric blends by atomic force microscopy," Journal of Polymer Science, Part B, vol. 44, no. 3, pp. 492-503, 2006.

[22] R. Y. F. Liu, Y. Jin, A. Hiltner, and E. Baer, "Probing nanoscale polymer interactions by forced-assembly," Macromolecular Rapid Communications, vol. 24, no. 16, pp. 943-948, 2003.

[23] M. S. Sanchez, J. M. Mateo, F. J. R. Colomer, and J. L. G. Ribelles, "Nanoindentation and tapping mode AFM study of phase separation in poly(ethyl acrylate-co-hydroxyethyl methacrylate) copolymer networks," European Polymer Journal, vol. 42, no. 6, pp. 1378-1383, 2006.

[24] S. N. Magonov, J. Cleveland, V. Elings, D. Denley, and M. H. Whangbo, "Tapping-mode atomic force microscopy study of the near-surface composition of a styrene-butadiene-styrene triblock copolymer film," Surface Science, vol. 389, no. 1-3, pp. 201-211, 1997. 

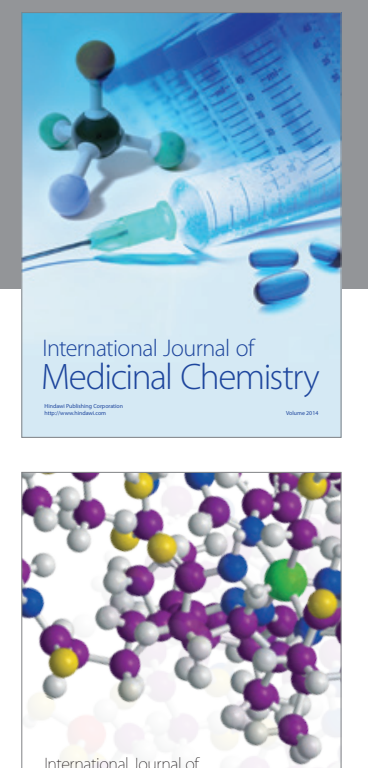

\section{Carbohydrate} Chemistry

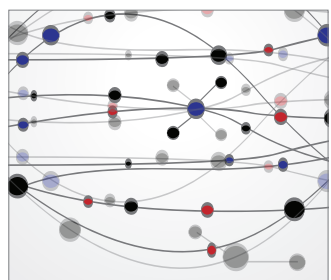

The Scientific World Journal
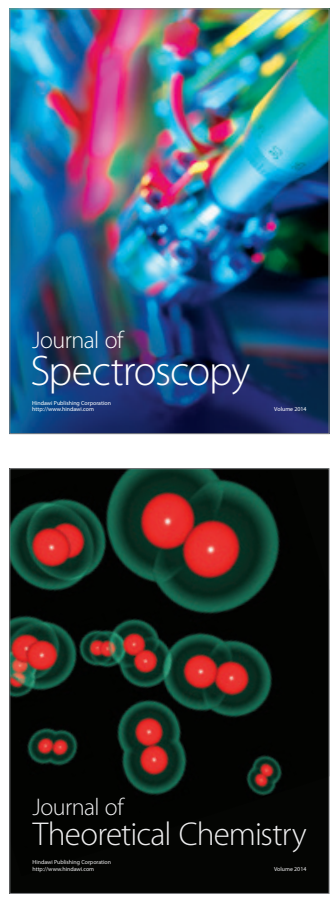
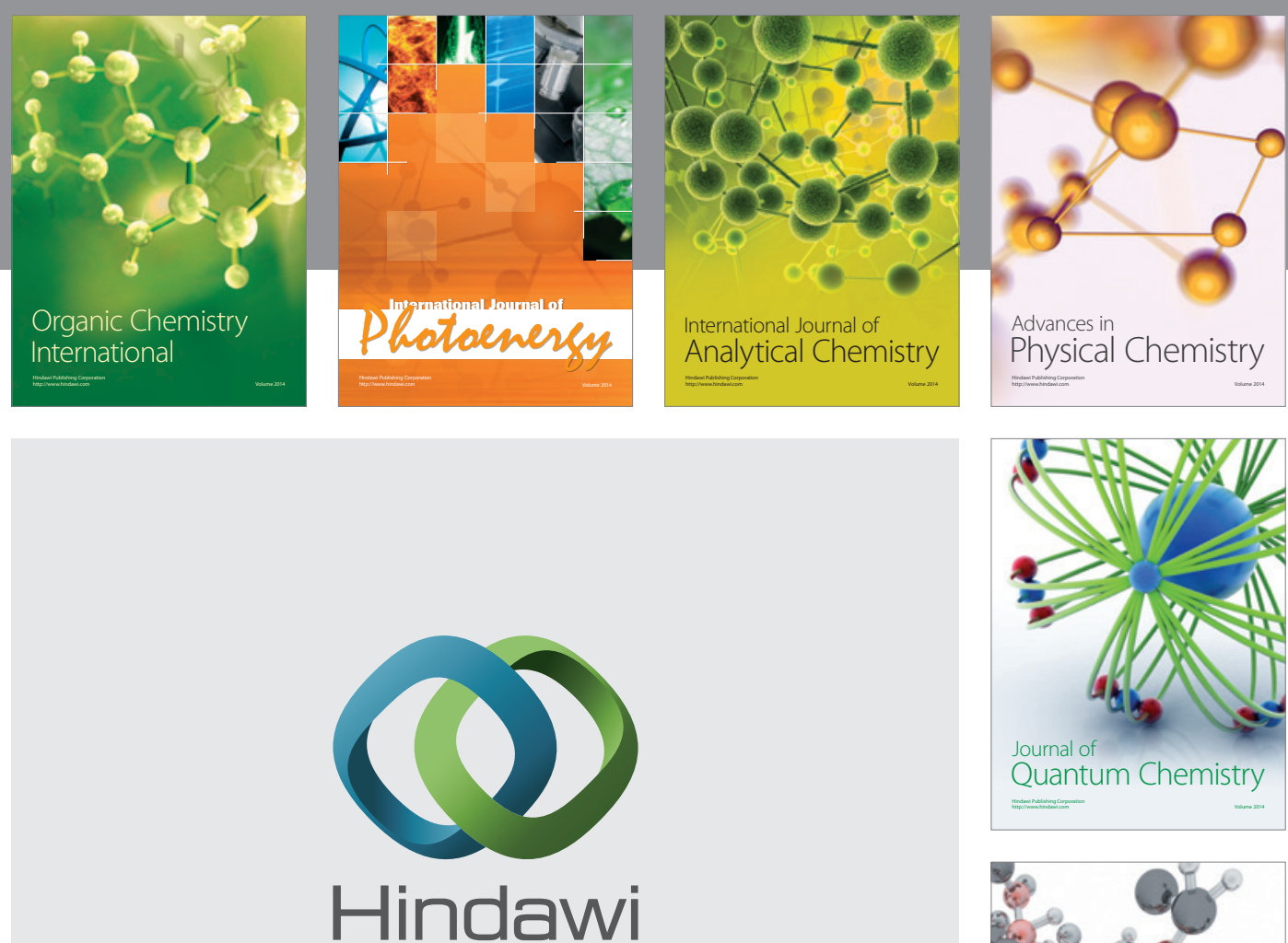

Submit your manuscripts at

http://www.hindawi.com

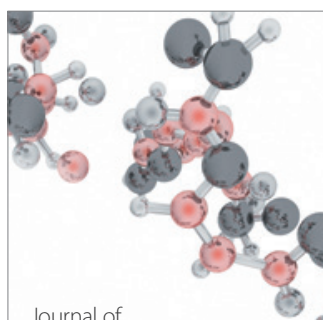

Analytical Methods

in Chemistry

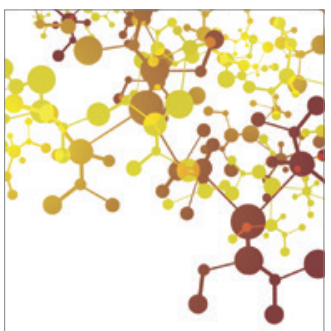

Journal of

Applied Chemistry

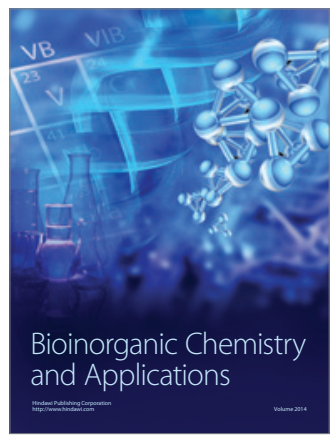

Inorganic Chemistry
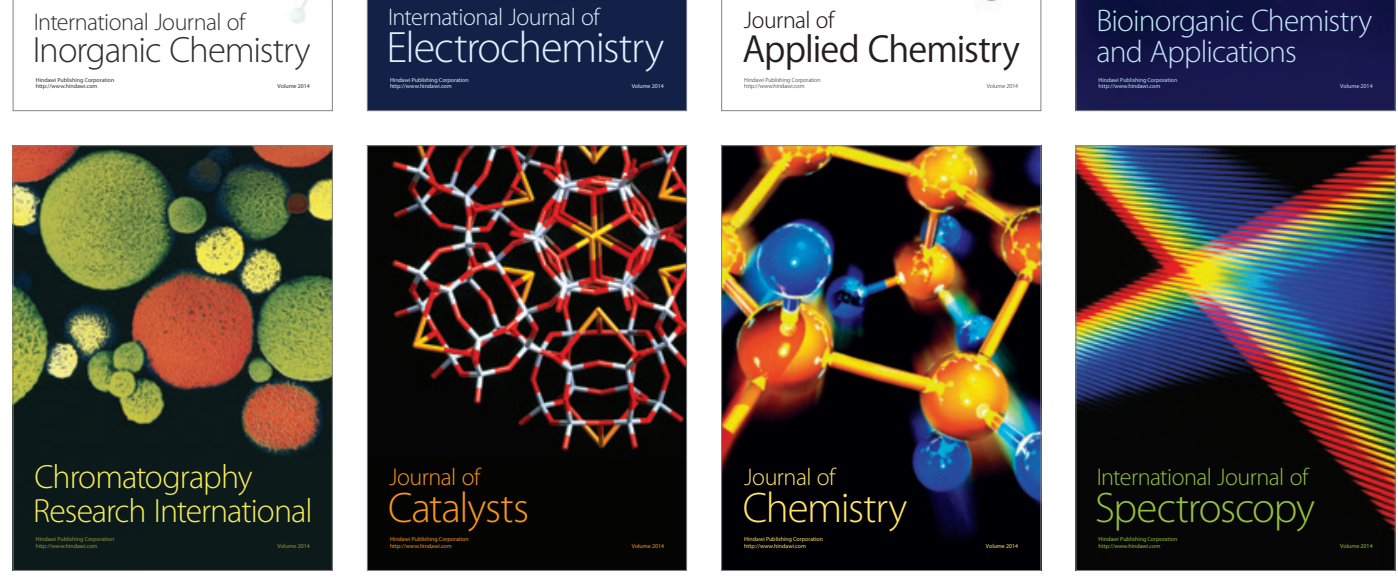